Aus dem pharmazeutischen. Institut der Universitat Berlin.

\title{
Der Alkaloidgehalt der Bilsenkrautblätter, der Tollkirschenblätter und ihrer Extralkto.
}

Von O. Anselmino.

(Eingegangen den 5. VIT. 1913.)

Das Deutsche Arzneibuch, 5. Ausgabe 1910, schreibt für Bilsenkrautblätter und für Tollkirschenblätter je einen auf Hyoscyamin berechneten Mindestgehalt an Alkaloiden vor und setzt ferner fest, daß Bilsenkrautextrakt und Tollkirschenextrakt auf einen bestimmten, gleichfalls auf Hyoseyamin berechneten Gehalt an Alkaloiden eingestellt werden.

Bei der Bereitung dieser Extrakte wird eine Ausbeute von wenigstens 20\% der angewandten Menge Blätter erzielt. Der Alkaloidgehalt in den Extrakten sollte somit, unter der Annahme, daß bei Innehaltung des vorgeschriebenen Auszugsverfahrens die Alkaloide aus den Blättern völlig in die Extraktbrühen übergehen, und daß bei dem Eindampfen keine Verluste entstehen, höchstens das Fünffache des Alkaloidgehaltes in den Blättern betragen.

Bei den vom Arzneibuch vorgeschriebenen Zahlen für Tollkirschenblätter und Tollkirschenextralkt trifft dieses Verhältnis zu, hier sollen die Blätter wenigstens $0,3 \%$ Alkaloide enthalten und das Tollkirschenextrakt das Fünffache, nämlich 1,5\%. Die Forderungen des Arzneibuches für die Bilsenkrautblätter und das Bilsenkrautextrakt dagegen stimmen mit dieser Ueberlegung nicht überein, denn die Blätter sollen mindestens $0,07 \%$ Alkaloide enthalten und das Extrakt 0,5\%, also nicht das Fünffache, sondern das Siebenfache. Diesem Verhältnis würde entweder eine Extraktausbeute von nur $14 \%$ entsprechen, oder, wenn die Blätter $0,07 \%$ enthalten, so könnte das Extrakt (bei 20\% Ausbeute) höchstens 0,35\% enthalten, oder drittens, wenn das Extrakt 0,5\% enthält, dann müßten in den Blättern wenigstens $0,1 \%$ Alkaloide enthalten sein.

Welche von diesen drei Möglichkeiten die richtige ist, müßte sich experimentell erweisen lassen. Es war somit zunächst nochmals zu untersuchen, in welcher Ausbeute ein vorschriftsmäBiges Extrakt gewonnen wird und dann, wie sich der Alkaloidgehalt der Blätter zu dem Alkaloidgehalt des Extraktes verhält, ob sich überhaupt aus Blättern mit dem vorgeschriebenen Mindestgehalt von 0,07\% 
Alkaloiden ein Extrakt mit 0,5\% Alkaloiden, wie es das Arzneibuch verlangt, herstellen läßt.

Es zeigte sich im Verlauf der Untersuchungen, daß eine Extraktausbeute von über $20 \%$ der angewandten Blätter erzielt wird; es zeigte sich aber auch, daß die Resultate der Alkaloidbestimmungen mit den vom Arzneibuch geforderten Zahlen übereinstimmten. Dieses vorschriftsmäßige Ergebnis stellt indessen eine Unmöglichkeit dar, denn es ist nicht wohl denkbar, daß in dem Extrakt mehr Alkaloide enthalten sein sollen, als überhaupt in den Blättern vorhanden waren. Es muß also entweder bei der Bestimmung der Alkaloide im Extrakt etwas als Alkaloid titriert werden, das kein Alkaloid ist, oder in den Blättern wird nach der Methode des Arzneibuchs nur ein Tejl der wirklich vorhandenen, d. h. in die Extraktflüssigkeiten übergehenden Alkaloide bestimmt, während der andere Teil sich aus irgend einem Grunde der Bestimmung entzieht.

Da nun von vomherein nicht anzunehmen ist, daß die in genau der gleichen Weise auszuführende Alkaloidbestimmung der Tollkirschenblätter andersartig verläuft als die der Bilsenkrautblätter, obwohl die Gehaltsforderungen des Arzneibuches darauf hindeuten, so war es zur Klärung der Frage erforderlich, Parallelversuche mit Bilsenkrautblättern und mit Tollkirschenblättern anzustellen.

Bei der Ausführung der experimentellen Arbeiten hatte ich mich der Unterstützung des Herrn cand. pharm. Karl M a y e r und ganz besonders des Herrn cand. pharm. $\mathrm{S}$. $\mathrm{N}$ e u s t ad t zu erfreuen.

Zunächst wurde von den Blättern, außer der Bestimmung des Feuchtigkeitsgehaltes und des Aschengehaltes, der Gehalt an Alkaloiden nach der Vorschrift des Arzneibuches ermittelt. Alsdann wurden die Blätter mit verdünntem Weingeist ausgezogen und von den beiden Auszügen (Auszug I und Auszug II) auß3er dem spezifischen Gewicht und dem Trockenrückstand, der Alkaloidgehalt bestimmt. Darauf wurden die beiden Auszüge vereinigt und das Extrakt hergestellt; nebenbei wurde die Menge des ausgeschiedenen Chlorophylls und der harzigen Bestandteile festgestellt. Von dem Extrakt endlich wurden die Ausbeute, der Feuchtigkeitsgehalt und der Alkaloidgehalt bestimmt.

\section{Hyoscyamus.}

1. Folia Hoscyami I.

a) Feuch tigkeitsgehalt:

$2,1080 \mathrm{~g}$ Blitter verloren beim Trocknen $0,2250 \mathrm{~g}$ an Gewicht $=10,67 \%$.

$1,9463 \mathrm{~g}$ Blätter verloren beim Trocknen 0,2065 g an Gewicht $=10,61 \%$. 
b) Aschengehalt :

$2,1080 \mathrm{~g}$ Blätter hinterließen 0,3828 g Asche $=18,21 \%$.

$1,9463 \mathrm{~g}$ Blättcr hi.tterließen 0,3581 g Asche $=18,40 \%$.

c) Alkuloidgehalt nach D. A.-B. 5:

Verbraucht wiurden $2,47 \mathrm{ccm} \mathrm{n/100} \mathrm{Salzäure}=0,071 \%$.

Verbraucht wurden $2,35 \mathrm{ccm} \mathrm{n} / 100$ Snlzsëure $=0,068 \%$.

Verbraucht wurden $2,48 \mathrm{~cm} \mathrm{~cm} / 100$ Salzsäure $=0,072 \%$.

2. Die Auszügeder Bilsenkratblätter I.

a) Vom Auszug $\mathrm{I}$ wurden erhalten aus $500 \mathrm{~g}$ Droge $=2380 \mathrm{~g}$.

Vom Alssug II wurden erhalten aus $500 \mathrm{~g}$ Droge $=1415 \mathrm{~g}$.

b) Spezifisches Gewicht (Pyknometer 18,50).

Der zum Ausziehen verwendete Spir. dil. 0,8921.

Auszug I 0,9112.

Auszug II 0,9011 .

c) Trockenrückstnnd:

Auszug I: je $24 \mathrm{~g}$ Flü-si $r$ lkeit ( $=5 \mathrm{~g}$. Droge) hinterlir:ßen

$1,043 \mathrm{~g}$ Trockenrückstand $=4,35 \%=$ anf Droge berechnet $20,86 \%$.

$1,030 \mathrm{~g}$ Trockenrückstand $=4,29 \%=$ auf Droge berechnet $20,60 \%$.

Ausqug II: je $28 \mathrm{~g}$ Flïssigkeit (=10 g Droge) hinterließen

$0,4598 \mathrm{~g}$ Trockenriickstand $=1,64 \%=$ uuf Droge borechnet $4,60 \%$.

$0,4518 \mathrm{~g}$ Trockenrückstund $=1,61 \%=$ auf Droge beruchnet $4,52 \%$.

Die durchschnittliche Summe der Trockenrückstände in beiden Auszügen beträgt demnach 25,3 v. H. der lufttrockenen Blätter.

d) Alkaloidgehult:

Auszug I: Angewandt wurden je $06 \mathrm{~g}$ Flüssigkeit (= $20 \mathrm{~g}$ Droge), die auf dem Wasserbade eingeengt und dann nach der Vorschrift des Arzneibuchs behandelt wurden.

Verbraucht wurden 4,67 ccin $n / 100$ Salzzäure $=0,021 \%=$ auf Droge berechnet $0,101 \%$.

Verbraucht wurden 4,48 cem n/100 Salzsäure $=0,020 \%=$ auf

Droge berechnet $0,097 \%$.

Auszug II: Angewandt wurden je 56,6 g Flüssigkeit ( $=20 \mathrm{~g}$ Droge).

Verbraucht wurden $0,49 \mathrm{ccm} \mathbf{n} / 1$ oo Salzsäure $=0,004 \%=$ auf Droge berechnet $0,011 \%$.

Verbraucht wurden $0,39 \mathrm{ccm} n / 100$ Salzsäure $=0,003 \%=$ auf Droge berechnet $0,009 \%$.

Die durchschnittliche Summe des Alkaloidgehalts in beiden Auszügen beträgt demnach 0,109 v. H. der lufttrockenen Blätter. 
3. Das Extrakt a s den BilsenkrautbläternI.

$1190 \mathrm{~g}$ Auszug I und $707 \mathrm{~g}$ Auszug II (= $250 \mathrm{~g}$ Droge) wurden zu Extractum spissum verarbeitet. Die Menge des ausgeschiedenen Chlorophylls usw. betrug $8 \mathrm{~g}$ (= $3 \%$ der Droge). An fertigem Extrakt wurden erhalten $55 \mathrm{~g}=22 \%$ der angewandten Droge.

a) Fouchtigkeitsgehalt:

$1,170 \mathrm{~g}$ Extrakt verloren beim Trocknen $0,189 \mathrm{~g}$ an Gewicht $=16,1 \%$.

b) Alkaloidgehalt nach D. A.-B. 5:

Vorbraucht wurden $3,41 \mathrm{ccm} n / 100$ Sulzsäuro $=0,493 \%=$ auf Droge berechnet $0,108 \%$.

Verbraucht wurden 3,51 com $n / 100$ Salzsäure $=0,507 \%=$ auf Droge berechnet $0,111 \%$.

\section{Folia H yos cyami II.}

Der Alkaloidgehalt der Blätter betrug 0,065\%, der Alkaloidgohalt der vereinigten Auszüge 0,104\%, das Extractum spissum ist bei der Fertigstellung verunglückt.

Es wurden noch weitere Proben von Bilsenkrautblättern untersucht, um noch weitere zahlenmäßige Belege für die Schlußfolgerung zu erhalten. Diese Untersuchungen lieferten aber ganz abweichende Resultate, was den Anstoß gab, die Bilsenkrautblätter des Handels einer genauen Prüfung zu unterwerfen. Die Ergebnisse sind in der folgenden Mitteilung niedergelegt.

\section{Belladonna.}

1. Folia Belladonnae.

a) Feuchtigkeitsgehalt:

4,92 g Blätter verloron beim 'Troaknen 0,385 g an Guwicht $=\mathbf{7 , 8} \%$.

$1,75 \mathrm{~g}$ Blätter verloren bein 'Trosknen $0,136 \mathrm{~g}$ an Gewicht $=7,8 \%$.

b) Aschengehalt:

$1,919 \mathrm{~g}$ Blätter hinterließen $0,259 \mathrm{~g}$ Ascho $=13,4 \%$.

$3,000 \mathrm{~g}$ Blätter hinterließen 0,409 o Asche $=13,6 \%$.

v) Nkaloidgehalt:

Verbraucht wurden $11,85 \mathrm{~cm} \mathrm{n} / 100$ Salzsäure $=0,341 \%$.

Verbraucht wurden $11,43 \mathrm{com} n / 100$ Salzsëure $=0,330 \%$.

2. Die Auszügeder Tollkirschenblät ter.

a) Vom Auszug I wurden erhalten aus $200 \mathrm{~g}$ Droge $930 \mathrm{~g}$.

Vom Auszug II wurden erhalten aus $200 \mathrm{~g}$ Droge $560 \mathrm{~g}$.

b) Spezifisches Gewicht:

Der zum Ausziehen vorwendete Spir. dil. 0,898.

Auszug I 0,916 .

Auszug II 0,905 . 
c) Trockenrückstand:

Auszug I: 25,365 g Flüssigkeit, hinterließen 0,934 g Trockenrückstand $=3,7 \%=$ auf Droge berechnet $17,1 \%$.

Auszug II: 22,557 of Flïssiskeit hinterließen 0,263 g Trockenrïckstand $=1,2 \%=$ auf Droge berechnet $3,3 \%$.

Die durchschnittliche Summe der Trockenrückstände in beiden Ausziigen beträgt demnach 20,4 v. H. der lufttrockenen Bliitter.

d) Alkaloidgehalt :

Auszing I: Angewandt wurden jo $93 \mathrm{~g}$ Flïsaigkeit ( $=20 \mathrm{~g}$ Droge $)$.

Verbraucht wurden $14,8 \mathrm{ccm} \mathrm{n} / \mathrm{1nn}$ Snlzä̈urn $=0,070 \%=$ auf Droge berceluet $0,321 \%$.

Verbraucht wurden $14,6 \mathrm{ccm} \mathrm{n} / \mathrm{nn}$ Snlzsäure $=0,068 \%=$ nuf Droge bereohnet $0,317 \%$.

Auszug II : Angewnndt wurden je $56 \mathrm{~g}$ Fliissigkeit ( $=20 \mathrm{~g}$ Drogc). Verbraucht wurden $1,1 \mathrm{ccm} \mathrm{n} / 100$ Sulzsiure $=0,008 \%=$ auf Droge berechnet $0,024 \%$.

Verbraucht wurden $1,2 \mathrm{ccm} n / 100$ Snlzsäure $=0,009 \%=a$ f Droge berechnet $0,026 \%$.

Bei der Aunlyso einer zweiten Extraktion verbrauchte die $20 \mathrm{~g}$ Droge entsprechende Menge des Gemisches dor Auszüge in zwei Ver-

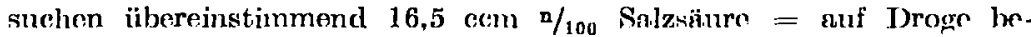
rechnet $0,357 \%$.

Die durchschnittliche Summe des Alkaloidgehalts in beiden Auszïgen beträgt demnach 0,344 v. H. der lufttrockenen Blätter.

3. I) as Gxtraktausden Tollkirschenblätern.

Aus $500 \mathrm{~g}$ Tolia wurden $3700 \mathrm{~g}$ Auszüge prhalten. Die Hälfte davon, $1850 \mathrm{~g}$ (=250 g Droge) wurden zu Nxtractum spissum verarbeitet. Die Menge des ausgeschiedenen Chlorophylls betrug $8 \mathrm{~g}(=3 \%$ der Bläter $)$. An fertigem lixtrakt wurden erhalten $53 \mathrm{~g}=21,2 \%$ der angewandton Droge.

a) Feuch tigkeitsgohult:

1,040 g Extrakt verloren beim Troeknen 0,136 $\mathrm{g}$ nn Grwicht. $=13,1 \%$.

b) Alkaloidgehalt:

$3,706 \mathrm{~g}$ Extrakt verbrauchton $12,9 \mathrm{~cm} \mathrm{n} / 100$ Snlasäurn $=1,52 \%$ $=$ auf Droge berexhnet $0,323 \%$.

$2,844 \mathrm{~g}$ Extrakt verbrauchten $10,2 \mathrm{cem} \mathrm{n} / 100$ Salzsïure $=1,56 \%$ $=$ auf Droge borechnet 0,330\%.

Das Ergebnis ist also kurz zusammengefaßt, jeweils auf Droge herechnet, folgendes:

B i ls on k ra u I: Dis Blätter enthielten (nach D. A.-B. 5) 0,07\% Alkaloide, die Auszïge zusamuen 0,108\%, das Extrakt 
0,109\%. Die Menge des Extraktes betrug 22\%, sein absoluter Alkaloidgehalt 0,5\%.

B i ls en k r a t II: Die Blätter enthielten 0,065\% Alkaloide, die Auszüge zusammen 0,104\%.

T o llki rsch e n: Die Blätter enthielten 0,335\% Alkaloide, die Auszüge zusammen 0,344\%, das Extrakt 0,327\%. Die Menge des Extraktes betrug 21\%, sein absoluter Alkaloidgehalt 1,54\%.

Die für die Blätter und die Extrakte gefundenen Alkaloidmengen stimmen also mit den vom Arzneibuch geforderten Zahlen überein. Bilsenkrautblätter mit 0,07\% Alkaloid gaben ein Extrakt mit $0,5 \%$, Tollkirschenblätter mit $0,3 \%$ ein Extrakt mit 1,5\%. Bezieht man jedoch den Alkaloidgehalt der Extrakte auf die Blätter, so kommen für Bilsenkrautblätter 0,109 statt 0,07\% heraus, während sich die Zahl für Tollkirschenblätter nicht erhöht.

Es war nun erforderlich zu untersuchen, ob durch doppelte Extraktion der Bilsenkrautblätter mit Aether derselbe Alkaloidgehalt wie in den weingeistigen Auszïgen gefunden wird.

Zunächst wurden die Blätter nach der Vorschrift des Arzneibuches behandelt, dabei wurden $2,48 \mathrm{ccm}{ }^{n} / 100$ Salzsäure verbraucht, was $0,0717 \%$ Alkaloid entspricht. Darauf wurden die Blätter mit dem Rest des Aethers und der zugesetzten Lauge auf dem Wasserbade erwärmt, bis aller Aether verdunstet war und darauf abermals mit Aether extrahiert. Bei der zweiten Titration wurden 2,2 ccm n/100 Salzsäure verbraucht. In dem Rückstande war, da nur die Hälfte des Aethers zur Titration verwendet wird, noch dieselbe Menge Alkaloid enthalten. Es war also in den Blättern eine Alkaloidmenge vorhanden, die entsprach 2,48 ccm (I. Titration) $+2,2 \mathrm{ccm}$ (II. Titration) $+2,2 \mathrm{ccm}$ (Rückstand) $=6,88 \mathrm{ccm}{ }^{\mathrm{n}} / 100$ Salzsïure oder $0,099 \%$ Alkaloide in den lufttrockenen Blättern.

Die vom Arzneibuch vorgeschriebene Methode für die Gehaltsbestimmung der Bilsenkrautblätter ergibt also nicht den wahren Gehalt an Alkaloid, sondern einen zu niedrigen Wert.

Während aus den Tollkirschenblättern durch verdünnten Weingeist dieselbe Menge Alkaloid ausgezogen wird (in Form von Alkaloidsalz) wie nach dem Versetzen mit Lauge durch Aether, gehen br:i den Bilsenkrautblättern mehr Alkaloide in den Weingeist über alk in den Aether, oder vielmehr es werden in den eingedampftern alkoholischen Auszügen mehr Alkaloide gefunden, als in der kalten Aetherausschüttelung. $\mathrm{Da}$ bei beiden Drogen das angewandte Verfahren vollkommen dasselbe ist, so kann der Grund des verschiedenen Verhaltens darin erbliekt werden, daß in den Bilsenkrautblättern ein anderes Alkaloidgemisch als in den Tollkirschen- 
blättern enthalten ist, oder noch wahrscheinlicher, daß die Art der Bindung der Alkaloide in beiden Drogen verschieden ist, daß ein Teil der Bilsenkrautalkaloide in einer Form vorliegt, die durch das Verfahren bei der Alkaloidbestimmung der Blätter nicht gespalten wird. Bei der äußerst geringen Menge Alkaloid, die in dem Bilsenkraut vorkommt, dürfte es wohl aussichtslos sein, einen exakten Nachweis dieser Vermutung zu erbringen, oder die Differenzierung der Alkaloidformen zu ermöglichen.

Dos Ergebnis der in der folgenden Mitteilung niedergelegten Untersuchungen läßt indes noch eine andere Deutung zu, insofern nämlich, als das Alkaloidgemisch, das nur teilweise in die kalte Aetherausschüttelung übergeht, nicht den Folia Hyoscyami, sondern der Herba Hyoscyami entstammt.

\title{
Die Bilsenkrautblätter des Handels.
}

\author{
Von O. Anselm in o und E. Gilg.
}

(Eingegangen den 5. VII. 1913.)

In vorstehender Mitteilung wurde gezeigt, daß die Extrakte der Bilsenkrautblätter mehr Alkaloide enthalten, als man auf Grund der Alkaloidbestimmung der Blätter erwarten kann.

Bei der Absicht, diesen Befund, abgesehen von den Zahlenangaben des Arzneibuches, durch mehr als zwei Beispiele zu stützen, orhielten wir bei Verwendung einer neuen Sendung Bilsenkrautblätter abweichende Resultate insofern, als die Alkaloidbestimmungen der Blätter, der Auszüge und der Extrakte Werte ergaben, die nicht mit denen der übrigen Bilsenkrautblätter und -Präparate übereinstimmten, sondern, die im Sinne der bei den Belladonnablïttern gemachten Erfahrungen verliefen.

Das fertige Extrakt unterschied sich von dem Bilsenkrautextrakt durch seine Farbe, die mehr rotbraun war, ganz besonders aber dadurch, daß sein Geruch von dem typischen Geruch des Bilsenkrautextraktes morklich abwich. Der auffallendste Unterschied zeigte sich aber beim Eintrocknen des Extraktes in dünner Schicht zum Zwecke der Bestimmung des Wassergehaltes. Während Bilsenkrautextrakt, das den Anforderungen des Arzneibuchs entspricht, sich dabei aufblähte und der graubraune Trockenrückstand eine ungleichmäßige Oberflïche zeigte, war der Rückstand des in 